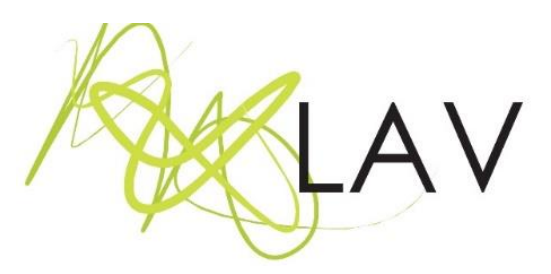

\title{
Oficios artesanales resignificados a través del proceso creativo con el lenguaje textil: una experiencia en la Educación Superior ${ }^{1}$
}

Ofícios artesanais resignificados através do processo criativo com a linguagem têxtil: uma experiência na Educação Superior

Handicrafts re-signified through the creative process with the textile language: an experience in Higher Education

\author{
Vanessa Freitagi \\ Universidad de Guanajuato \\ Jorge Alberto Custodio Casimiroii \\ Universidad de Guanajuato
}

\begin{abstract}
Resumo
Este artigo pretende explorar as possibilidades criativas de estudantes universitários através do uso e prática da linguagem têxtil. Se busca a ressignificação de miradas sobre a arte popular mexicana e em particular, sobre as criações têxtis tradicionais e contemporâneas. Utilizamos a perspectiva teórico-metodológica da IBA (Investigación Basada en las Artes) para fundamentar nosso processo de trabalho com os estudantes. Entre os resultados do estudo, encontramos que o fazer manual, as práticas artesanais e de ofícios contribuem a conscientizar e a construir um olhar crítico sobre o valor dessas atividades na vida cotidiana, e também, a considerarlas como uma experiência artística e criadora por si mesma. Além disso, desenvolvem nos estudantes algunas atitudes como a paciência, o trabalho coletivo, a concentração e a capacidade de reconhecer distintas estéticas da linguagem têxtil.
\end{abstract}

Palavras-chave: oficios artesanais, processos criativos, arte textil.

\section{Abstract}

This paper intends to explore the creative possibilities of university students through the use and practice of the textile language. It seeks the resignification of views on Mexican popular art and, in particular, on traditional and contemporary textile creations. We explore the perspective of the ABR (Arts Based Research) as the basis for our work process with the students. Among the results of the study, we find that manual making, craft and handmade practices contribute to raising awareness and constructing a critical view on the value of these activities in everyday life as well as considering them as an artistic and creative experience in itself. In addition, they develop in students some attitudes such as

\footnotetext{
${ }^{1}$ Resultado parcial del proyecto de investigación financiado en la Convocatoria Interinstitucional de Investigación Científica, 2018.
}

Revista Digital do LAV - Santa Maria - vol. 12, n. 1, p. 38 - 54 - jan./abr. 2019 ISSN 1983 - 7348 http://dx.doi.org/10.5902/1983734837114 
patience, collective work, concentration and the ability to recognize distinct aesthetics of the textile language.

Keywords: handicraft, creative process, textile art.

\section{Introducción}

La presente propuesta busca discutir la importancia de construir miradas más significativas e incluyentes en torno a los oficios artesanales tomando como punto de partida, la experiencia práctica con el lenguaje textil, tanto el tradicional como el contemporáneo, en el contexto universitario. La base empírica de este estudio se fundamenta en las clases de telares impartidas en la Licenciatura en Cultura y Arte de la Universidad de Guanajuato, Campus León, que forma a gestores culturales en dicha ciudad. Desde 2013, hemos trabajado con pequeños grupos de estudiantes de la licenciatura, donde se han abordado temáticas relacionadas con el quehacer artesanal y artístico a través del lenguaje textil.

Nuestro objetivo fue resignificar los modos de concebir el oficio artesanal para la formación de los futuros gestores culturales. Es muy frecuente percibir de los y las estudiantes su desinterés o desconocimiento de los oficios artesanales y, en particular, las artesanías. Los oficios artesanales constituyen un conjunto de saberes tradicionales y formas de hacer objetos o cosas manualmente, es decir, panadería, sastrería, zapatería, entre otros. Las artesanías, por su parte son los productos del oficio artesanal, es decir, aquellos objetos que suelen ser comercializados, consumidos o usados por particulares. Tanto los oficios como las artesanías pueden ser dignos de estudio, análisis y temáticas para proyectos culturales. En las y los estudiantes se comparte una visión de que las artesanías son objetos ordinarios y que no hay un problema de fondo que las torne en un objeto de estudio. Finalmente, se ha reproducido un discurso que descalifica, invisibiliza o desconoce la artesanía como 'arte' (GREENHALGH, 1997), lo que contribuye en parte a no ubicarla dentro de sus campos de interés laboral o investigativo. Pensamos que esta forma de ver a las artesanías amerita un proceso de resignificación mediada por la práctica textil.

Como estrategia para contrarrestar esa visión normalizada sobre las prácticas, procesos y productos artesanales, buscamos a través de la docencia, problematizar la idea misma de oficios y de artesanías. Al mismo tiempo, nos interesa ofrecer una visión ampliada de lo que consideramos arte textil. Entendemos que es en el espacio del salón de clases donde podemos conocer las distintas posturas y visiones de mundo construidas por los y las estudiantes, así como, desestabilizar algunas verdades incorporadas por nosotros de 
manera inconsciente (CHALMERS, 2003), tales como, el tejido como una actividad asociada a lo femenino, y por ende, al contexto doméstico.

La licenciatura presenta una amplia oferta de talleres optativos que son todos muy atractivos y están abiertos a todos los y las estudiantes. El Taller de Telares suele tener más público femenino que masculino, aún cuando la carrera presenta un promedio de $60 \%$ de estudiantes mujeres y $40 \%$ de estudiantes varones ${ }^{2}$. Consideramos que el bajo interés de los varones se debe a que aún se asocia el tejido con una actividad "aburrida" y a la vez, "femenina", pero no siempre están conscientes de los estereotipos relacionados con quehacer textil. Por otro lado, muchas de los y las estudiantes que se interesaron por el taller fue porque ya habían tenido alguna experiencia previa en sus hogares con la práctica del croché o bordado (más recurrente con las estudiantes), o porque visitaron el taller semestres anteriores y observaron de qué se trataba el curso. Los y las estudiantes vieron que podrían aprender a hacer cosas tejidas (como bufandas) para sí mismas o para alguien más. También que podrían realizar mandalas, joyería textil, tapetes, alfombras, llaveros, adornos de pared, entre otros. Alguna de estas opciones, les motivaba más para tomar el curso.

Hemos atendido a grupos de estudiantes en un taller optativo de 4 horas semanales donde aprendían los principios básicos del tejido en bastidor de clavos. Los grupos suelen ser pequeños: entre 15 a 20 personas por semestre, mayoritariamente mujeres. Esto facilita un proceso de enseñanza-aprendizaje que genere una atención más individualizada a cada estudiante que lo curse. Durante las sesiones, conocen además del telar de clavos y otras técnicas textiles, como el bordado, el tejido de ganchillo y la costura. Paulatinamente, han podido experimentar con materiales reciclados (plástico, piel, trapos, cordones, entre otros), de acuerdo con su interés y propuesta de trabajo personal. Se alienta a que empiecen a trabajar con técnicas donde sientan curiosidad por aprender y a la vez, que investiguen qué quieren hacer (si será un adorno de pared, un arte-objeto o algo para usar/vestir). Como mediadores de ese proceso, ofrecemos posibilidades de trabajo mediante la adquisición de técnicas y la identificación visual de las características de cada rama textil, bien como, sus posibilidades creativas. Nos interesa que experimenten libremente, que planeen qué desean hacer, que piensen sobre los materiales a usar y los significados a plasmar en el objeto. No nos interesa tanto, aunque tampoco lo prohibimos, que lleguen con diseños y patrones textiles predeterminados, por un ejemplo. Alentamos que cada estudiante confeccione, borde, teja, cree su propio trabajo con un diseño

\footnotetext{
${ }^{2}$ Información solicitada a la coordinación del programa educativo.
} 
igualmente personalizado. Enseñamos trabajos de artistas textiles, de artesanos y artesanas que tejen de forma tradicional, o de creadores y creadoras que dialogan entre lo considerado tradicional y contemporáneo, artístico o artesanal, en sus producciones textiles. Fomentamos una mirada intercultural mediada por la práctica textil, lo que contribuye a ampliar sus repertorios visuales con referencias del mundo textil.

Tomando en cuenta que las dinámicas cambian cada semestre, hemos podido mantener algunas rutinas de trabajo donde, primeramente, las y los estudiantes conocen algunas técnicas sencillas dentro del lenguaje textil y buscan dominarlas al punto de permitirles pensar una propuesta con temática más personalizada. Posteriormente, investigan sobre su tema elegido - especialmente, a otros artistas, así como artesanos y artesanas - y a la vez, profundizan en el dominio de una técnica textil que desean conocer y aplicar en su proyecto. El proceso de conocer y 'adoptar' como influencia o inspiración a mujeres y hombres dedicados al arte o a la artesanía textil es significativo dado que es cuando conocen su historia y sus procesos creativos, pueden analizar la estética de sus trabajos. Es decir, encuentran en estas referencias tanto técnicas como temáticas para desarrollar su propuesta personal. Este proceso contribuye a paliar la ansiedad que sienten cuando incursionan al taller, dado que suelen decir que 'no saben qué hacer' o que 'no tienen idea de lo que quieren desarrollar' por la falta de referentes visuales en su cotidianidad. Conocer las propuestas de hombres y mujeres artistas y artesanos textiles no solo de México, sino de otros contextos, contribuye a ampliar el panorama de lo que se puede hacer y pensar con el lenguaje textil.

A la vez, en las clases problematizamos la relación dicotómica e históricamente construida de los conceptos de arte-artesanías o de artista-artesano (BOVÍSIO, 2002). Dichos conceptos surgen de la práctica misma del arte textil, y del conocimiento que adquieren los y las estudiantes con el trabajo de creadoras y creadores en este campo. Es importante mencionar que hay semestres en los que hemos solicitado a los y las estudiantes la adquisición y uso de un diario creativo personal. Paulatinamente, hemos observado que la mayoría que lo adopta, ha tenido un poco más claridad sobre lo qué quieren plasmar en sus proyectos textiles. Además, en cada sesión del taller se dejaban actividades muy concretas para que se registrara en sus diarios y finalmente, para que elaboraran bocetos de sus futuros proyectos textiles, reflexiones de sus propuestas y apuntes de clase.

Nosotros como docentes, realizamos un exhaustivo registro fotográfico del proceso de cada estudiante y aplicamos cuestionarios para evaluar sus percepciones sobre sus procesos de aprendizaje en el lenguaje textil, sobre arte y cultura popular, así como, las miradas 
construidas a partir su experiencia en el taller de telares. Compartiremos algunas de ellas en este texto y además, de nuestra perspectiva teórico-metodológica.

\section{Abordando el arte textil en la universidad: una experiencia en el Taller de Telares}

Solemos decir a los y las estudiantes universitarios que, salvo algunas excepciones, lo más probable es que nuestra relación con el tema artesanal haya empezado desde la infancia. Es decir, que algunos de nosotros y nosotras hayamos crecido en un ambiente familiar donde ya sea la madre o la abuela tejía, bordaba o costuraba en su tiempo libre. Es cuando problematizamos la idea misma generalizada de que el tejido es un trabajo exclusivamente femenino.

Si no a través del tejido, posiblemente tengan conocimiento de algún familiar que haya trabajado en oficios, tales como la panadería, la carpintería, la zapatería, por mencionar algunos. Sobre la práctica de los oficios en el ambiente familiar es frecuente, además, conocer la visión de las nuevas generaciones, especialmente en este contexto, quienes no se interesan por aprenderlo, y menos aún, en apoyar a su familia en el desarrollo de estas actividades, de acuerdo con algunos estudios sobre el tema (NOVELO, 2009; TUROK, 2015; DEL CARPIO, 2012; FREITAG, 2012).

A los y las jóvenes que llegan a la Universidad, tampoco se les incentiva el acercamiento a las artes y/o a las artesanías, mucho menos, a los oficios artesanales. Aun cuando se habla del tema en el contexto educativo, especialmente en las escuelas de artes en las que nos hemos formado, se transmite la idea de que la artesanía no es arte ya que consiste sólo en la realización de manualidades. Por otro lado, está la concepción de que el arte popular es lo que hacen personas sin formación artística. Con esto queremos decir que tanto en el ambiente familiar, como en el contexto educativo, no se visibilizan las prácticas artesanales y a sus creadores o creadoras como dignos portadores de un conocimiento para ser aprendido, apreciado y valorado socialmente también como arte.

Desde 2010 nos hemos dedicado al estudio de los oficios y del trabajo artesanal en México, especialmente, en algunas comunidades artesanales de Jalisco y de Michoacán. Este proceso nos ha llevado a conocer un amplio corpus de investigaciones dedicadas a analizar, entender, explicar y/o a promover las artesanías, los artesanos y las artesanas así como sus problemáticas en el contexto mexicano. Comprendimos entonces, que las artesanías no son solamente 'manualidades', especialmente cuando realizamos estudios para conocer las trayectorias de vida y de trabajo de artesanos de las localidades de Tonalá, Jalisco (FREITAG, 2012) y de Cuanajo, Michoacán (CASIMIRO, 2012). Estas experiencias han cambiado profundamente nuestra forma de ver y pensar las artesanías en lo general y 
especialmente, en este país. ¿Pero cómo construir esta mirada transformadora en el contexto universitario? Esta es nuestra constante búsqueda como docentes e investigadores.

Pensamos que en caso de que el gusto por el arte popular y por las artesanías no haya sido incorporado en la infancia a través del contexto familiar, la escuela sería entonces uno de los espacios para aprender a reconectarnos con nuestras tradiciones y culturas populares (BARBOSA, 2009). O en el último caso, que el encuentro con la cultura popular sea fomentado en el contexto universitario, tal y como pasa en instituciones educativas no formales como lo son los museos y los centros culturales.

En este texto, sistematizamos el trabajo realizado con estudiantes de la Licenciatura en Cultura y Arte. Los datos que se presentan a continuación son algunos fragmentos de información recabada a través de nuestra experiencia docente en las clases. Toda la información sobre los procesos de aprendizaje de los y las estudiantes la hemos registrado en un diario de clase personal, es decir, en el diario del profesor y la profesora; luego, aplicamos un cuestionario a los y las estudiantes al principio y al final de las clases, donde conocimos tanto el interés y las expectativas de cada con la materia cursada, como los logros y conocimientos adquiridos. En este último, las y los estudiantes también evaluaron las limitaciones enfrentadas y aquellos aspectos que hubieran querido estudiar en cada una de las sesiones. De igual manera, hemos realizado trabajo práctico (los proyectos textiles) como forma de plasmar ideas y detonar nuevas reflexiones estéticas y creativas sobre el lenguaje textil. Finalmente, hemos registrado los productos creados por las y los estudiantes a fin de conocer sus experimentos estéticos, el dominio técnico y descubrimientos tenidos en ese proceso.

Entendemos que a esta modalidad de investigación que considera el espacio educativo como un lugar donde se investiga y se aprende a través del arte, se conoce como investigación basada en el arte. En palabras de Ricardo Marín Viadel (2011) "se configura como un territorio de investigación con su propia identidad distintiva, situado justo en la intersección entre los problemas de las artes visuales y los problemas educativos" (p. 272).

Pensamos que la educación a través del arte, y en nuestro caso, a través del pensar y hacer arte textil, posibilita que puertas se abran a otras posibilidades de pensamiento y a otras formas de conocer el mundo mismo (LANAU Y PASCUAL, 2018). Desde esta perspectiva epistemológica, el pensamiento construye realidades y mediante el pensar artístico, también podemos transformar a la misma realidad. La Investigación Basada en las Artes (IBA) propone una confrontación crítica hacia los modelos tradicionales de 
enseñanza que buscan la formación de estudiantes pasivos, acríticos y domesticados (LANAU Y PASCUAL, 2018). Sino que retoma el valor de la intuición y de la experiencia como forma de acercarse al conocimiento (porque no solo hay una forma). Dice Hernández (2008, p. 90) que "la forma genuina de experiencia es la artística".

La IBA es un tipo de investigación de orientación cualitativa y su característica principal, es que utiliza procedimientos artísticos (literarios, visuales, musicales, performativos) para explorar la construcción de conocimientos con base en la práctica, en la experiencia y en la reflexión crítica (HERNÁNDEZ, 2008). Es, por lo tanto, una forma alternativa de investigación que explora tanto recursos emocionales como cognitivos en su puesta en práctica.

Consideramos a esta metodología la idónea para trabajar procesos creativos en el lenguaje textil. Para tal, hemos empezado el proceso con la experimentación práctica y el aprendizaje paulatino de saberes y técnicas propias de los textiles; se problematizó sobre la importancia de conocer cómo se hace un textil, qué implicaciones tiene el dominio de una técnica y el valor que deberíamos de imprimir a los productos, oficios y prácticas que tienen lo manual su principal característica. Los procesos creativos fueron fomentados a partir de la experiencia personal de las y los estudiantes con dicho lenguaje, donde testimonios biográficos han sido importantes para generar empatía, conexión y aprecio hacia el trabajo manual y el textil en particular. Un poco del trabajo realizado en el taller se describe a continuación.

\section{Aprender a reconocer y a resignificar el arte textil: una propuesta educativa}

Como responsables de la asignatura Taller de Telares, nuestro propósito fue trabajar la revalorización de los oficios artesanales a través de la experiencia directa e investigativa con el arte textil. En cuanto a los aspectos pedagógicos de la disciplina, hemos propuesto, que los y las estudiantes se familiaricen gradualmente con el lenguaje propio de la práctica textil, incluyendo sus principios técnicos y vocabulario, mismos que se usarían en el transcurso de las clases. De igual manera, se les incentivó la construcción de sus propios bastidores de clavo, dado que no tenemos infraestructura para enseñar el telar de pedal o de mesa. El telar de clavos ha sido la alternativa para dar a conocer los principios básicos del tejido. Es decir, las clases se enfocaron en la sensibilización de los y las estudiantes a través de texturas de los estambres, formas y/o materiales utilizados para tejer. El enfoque en el tejido consistiría en la aplicación de técnicas mixtas y con una estética contemporánea.

Revista Digital do LAV - Santa Maria - vol. 12, n. 1, p. 38 - 54 - jan./abr. 2019 ISSN 1983 - 7348 http://dx.doi.org/10.5902/1983734837114 
En un primer momento, el dominio de la técnica fue una prioridad de las clases, puesto que solo así, ellas y ellos podrían crear y usar una variedad de materiales para producir sus piezas. Las sesiones fueron divididas en cuatro módulos a lo largo del semestre: 1) Introducción al lenguaje textil y herramientas técnicas; 2) Arte textil mexicano; 3) Artesanas y artesanos textiles; 4) Artistas textiles de otros continentes. En cada módulo, se hacía una introducción teórica, acompañada de lecturas específicas sobre diferentes tipos de posibilidades técnicas y expresivas en el telar. También se compartía ejemplos de artistas, artesanas y artesanos que se dedicaban a explorar el lenguaje textil, así como el contexto de producción de algunos y algunas artistas textiles en México. Las clases eran complementadas con la exploración de materiales para tejer, de puntos, patrones y bocetos de proyectos textiles de manera individualizada. A través de la consulta de materiales bibliográficos y de páginas como el "Pinterest", "Instragram", "Facebook", blogs de artistas textiles de otros países y de México, y libros especializados, los y las estudiantes buscaban referencias visuales para sus propias propuestas artísticas.

Con el propósito de conocer cuáles eran las expectativas iniciales de los y las estudiantes antes de empezar el taller y qué significó al concluir las clases, aplicamos un breve cuestionario de respuestas abiertas y anónimas, donde pudiesen evaluar su desempeño académico y reflexionar sobre los conocimientos, habilidades y actitudes aprendidas a lo largo del curso. Citamos algunos testimonios:

El taller de textiles siempre llamó mucho mi atención, me parece una actividad realmente artística los trabajos hechos con ésta técnica. Mis expectativas siempre fueron poder aprender a realizar esta técnica con mis propias manos, la verdad no pensé aprender a hacer más de una técnica, cosa que así fue, puesto que realicé más de 5 trabajos con técnicas mixtas. (Comunicación personal, estudiante 1, 12 de noviembre de 2014)

Antes de comenzar el taller tenía una idea sobre lo que se iba a hacer, sabía que quería aprender y que iba a servirme como un ejercicio de relajación y concentración. Después del curso puedo decir que definitivamente el taller superó mis expectativas, no sólo me llevó los conocimientos compartidos, sino que también me llevó a una manera diferente de ver las cosas y una manera distinta de vivir. Aprendí mucho más de lo que esperaba y, no sólo eso, pude hacer una introspección sobre lo que sucedía en mí; la paciencia es algo que me llevo y el aprender el valor de las cosas. (Comunicación personal, estudiante 2, 23 de noviembre de 2015).

Al comenzar el curso mis expectativas eran bajas, ya que no estaba muy segura de que tipo de técnicas utilizaríamos en el taller o que tan complicadas serían y conforme se fue

Revista Digital do LAV - Santa Maria - vol. 12, n. 1, p. 38 - 54 - jan./abr. 2019 ISSN 1983 - 7348 http://dx.doi.org/10.5902/1983734837114 
desarrollando el semestre, me sentí muy conforme y feliz con las técnicas utilizadas en el mismo, ya que no solo desarrollábamos las técnicas de telar, sino nuestra propia creatividad vaciada en el telar. (Comunicación personal, estudiante 3, 14 de noviembre de 2016).

En semestres recientes (del 2016 al 2018), y a solicitud de los y las estudiantes, hemos incorporando otras técnicas textiles, como el tejido con ganchillo, el bordado, la costura, la arpillería ${ }^{3}$, el macramé y el tejido con materiales alternativos. Quizás por esta razón, se ha venido incrementando el público interesado en el taller, aunque sigue siendo un reto animar a los varones a tejer.

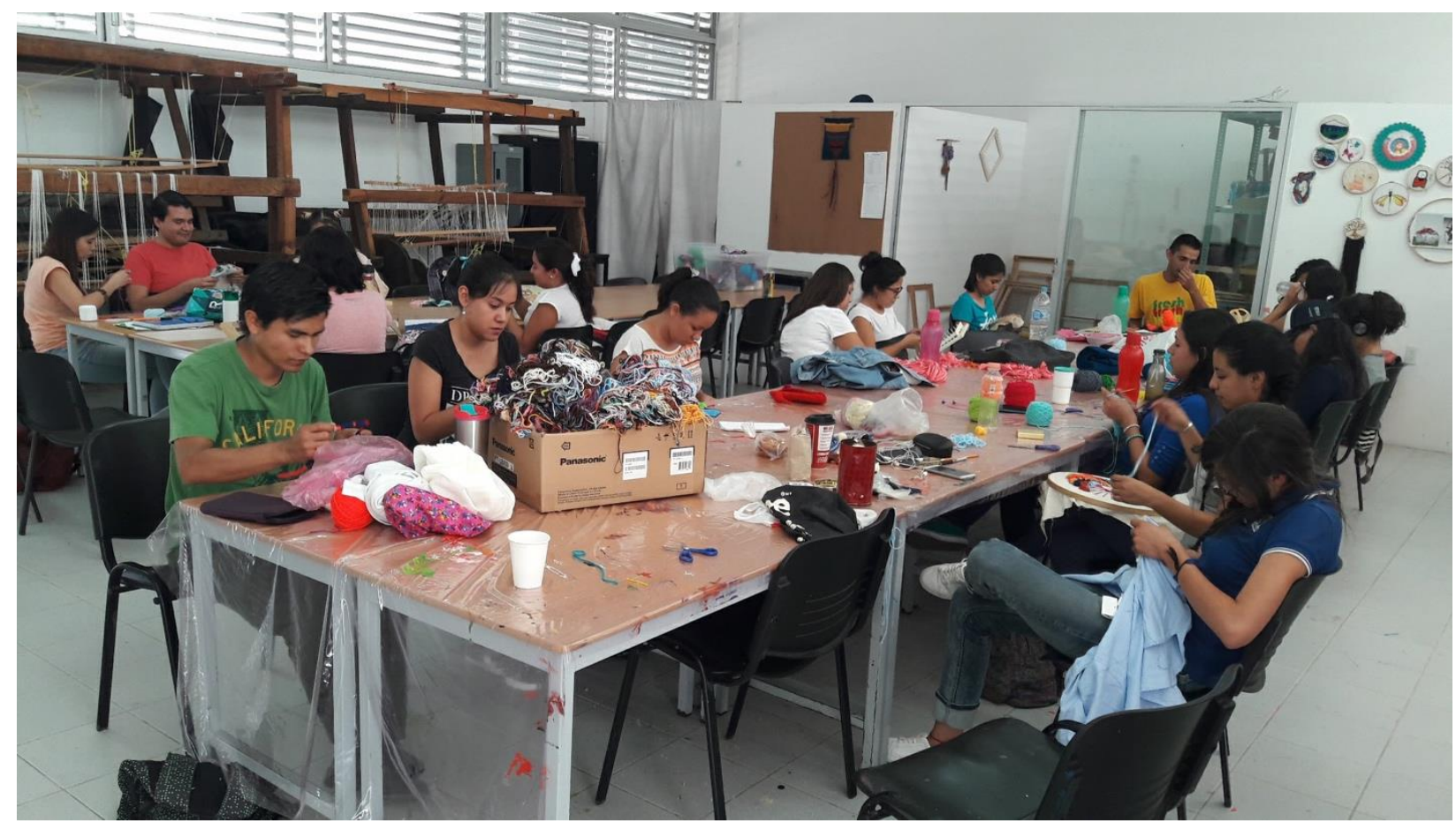

Figura 01. Estudiantes tejiendo en el Taller de Telares.

Fuente: archivo personal de los investigadores, 2017.

\footnotetext{
${ }^{3}$ Se trata de un tipo de bordado sobre yute ampliamente trabajado en Sudamérica, especialmente en Chile, cuya pionera ha sido Violeta Parra.
}

Revista Digital do LAV - Santa Maria - vol. 12, n. 1, p. 38 - 54 - jan./abr. 2019 ISSN 1983 - 7348 http://dx.doi.org/10.5902/1983734837114 


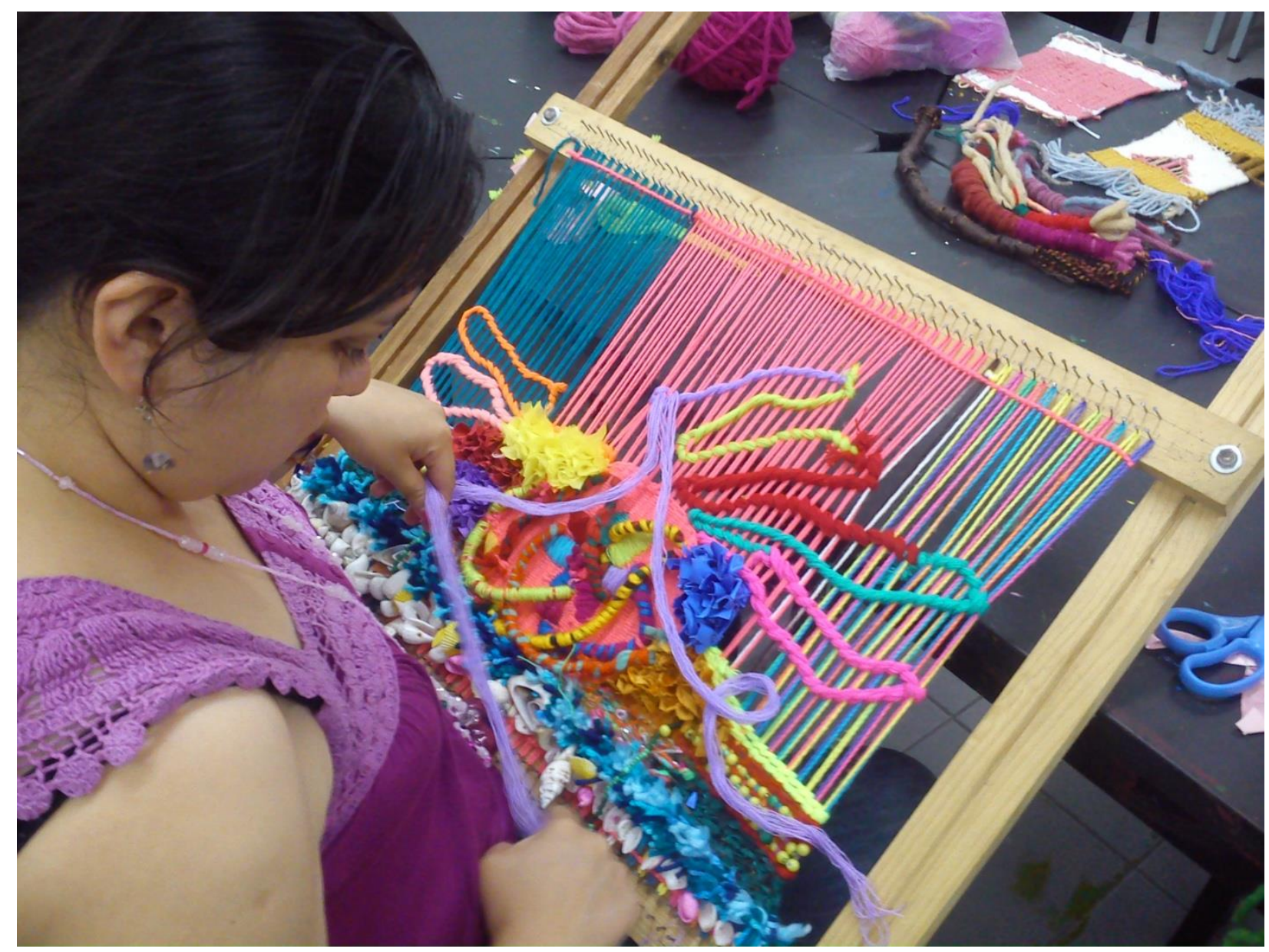

Figura 02. Estudiante trabajando en su proyecto textil.

Fuente: archivo personal de los investigadores, 2015.

Uno de los grandes desafíos de las clases de telares es enseñar la paciencia y la capacidad de observación del tejido, así como de visualización para diseñar sus propios proyectos. Generalmente, los y las estudiantes empiezan de forma intuitiva y realizan propuestas de manera muy espontáneas. No obstante, se muestran muy exigentes sobre aquello que producen y se inquietan con mucha facilidad. Otras veces, se frustran frente a un trabajo que no quedó bien resuelto técnicamente. Como mediadores de este proceso de aprendizaje, necesitamos estar atentos a las señales de desánimo, frustración o impaciencia, dado que el aprendizaje del oficio requiere de mucho tiempo y práctica constante. Hay veces que se toma todo un semestre aprender estas actitudes.

En estos momentos, solemos decirles que el tiempo que un maestro o maestra artesana requiere para realizar sus trabajos a la perfección es de toda una vida. Por lo que estos primeros pasos en el aprendizaje de los textiles deberían impulsar a ver el oficio desde otra perspectiva, desde un ritmo más lento. De esta manera, podrán apreciar y a valorizar

Revista Digital do LAV - Santa Maria - vol. 12, n. 1, p. 38 - 54 - jan./abr. 2019 ISSN 1983 - 7348 http://dx.doi.org/10.5902/1983734837114 
las cosas hechas a mano, los pormenores y las peculiaridades de los materiales usados por los artesanos. Lo podemos observar en el testimonio de esta estudiante:

Aprendí a ser paciente, observadora. A valorar el trabajo de este tipo, saber todo el proceso, tiempo, material y esfuerzo que representa; es toda una manera de comunicarte, por este medio puedes transmitir muchas cosas y también muchas otras cosas sobre nuestra cultura se ven reflejadas en estos trabajos. Me encantó poder tener la experiencia de ver de cerca el proceso de un telar de cintura, el trabajo y el ingenio que conlleva realizar una sola pieza de esta manera. Y no sólo eso, sino que no se toma en cuenta que al nosotros apoyar este tipo de manifestaciones culturales estamos rescatando parte de nuestras tradiciones y la esencia de nuestro pueblo. (Comunicación personal, estudiante 3, 20 de noviembre de 2013).

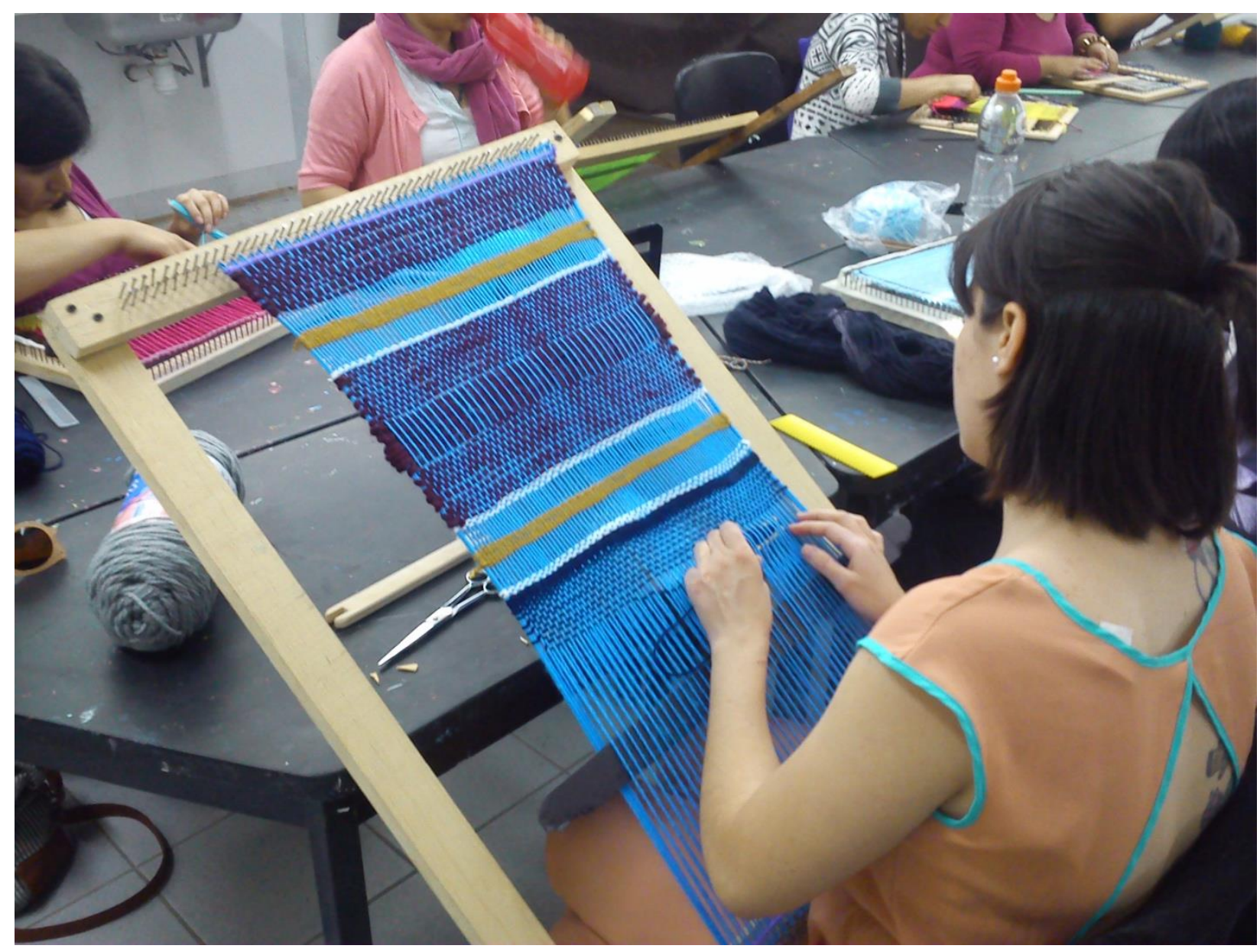

Figura 03. Alumna trabajando en su telar de clavos.

Fuente: archivo personal de los investigadores, 2014. 


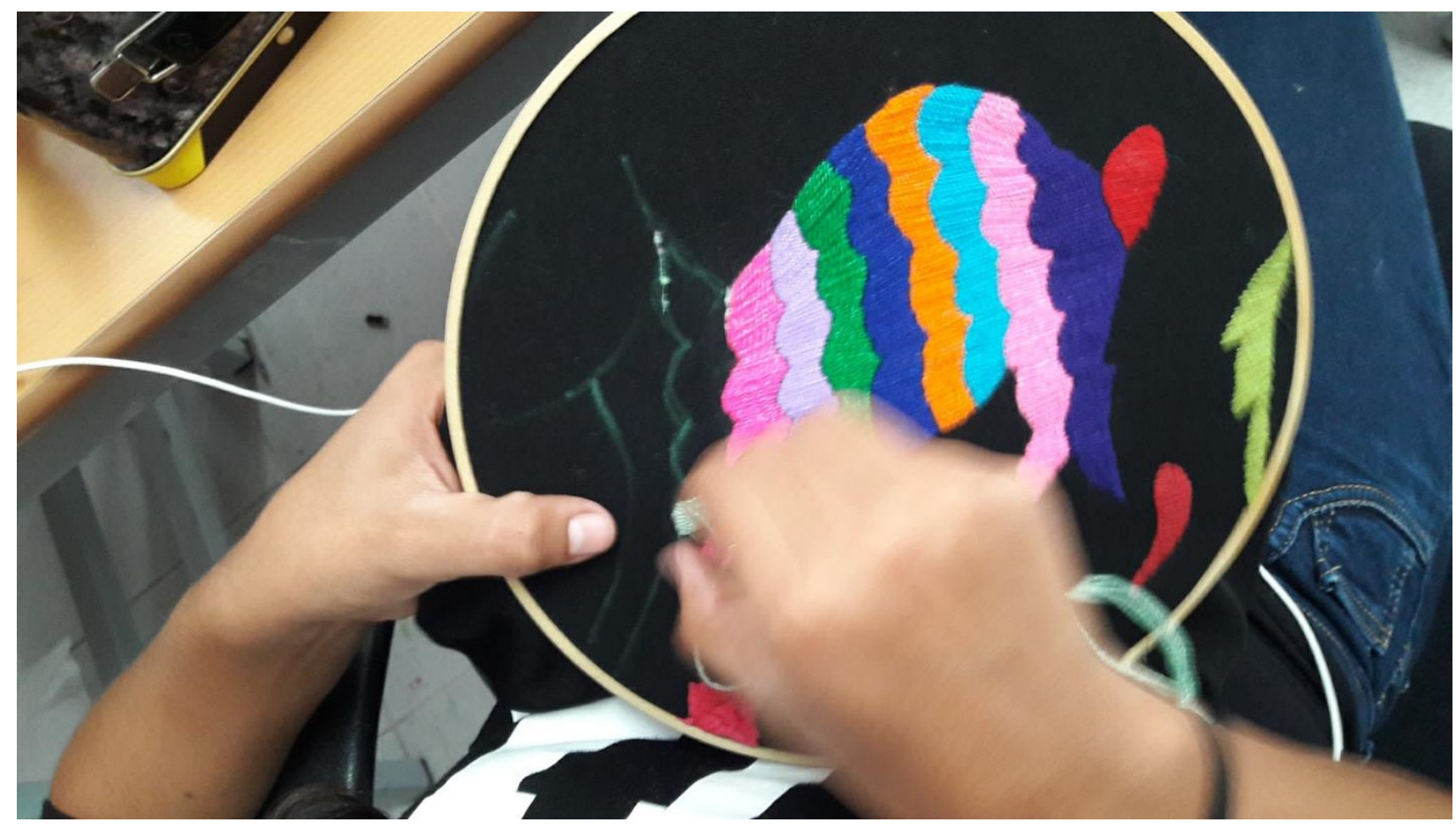

Figura 04. Bordado de alumna, inspirado en el bordado de Tenango.

Fuente: archivo personal de los investigadores, 2016.

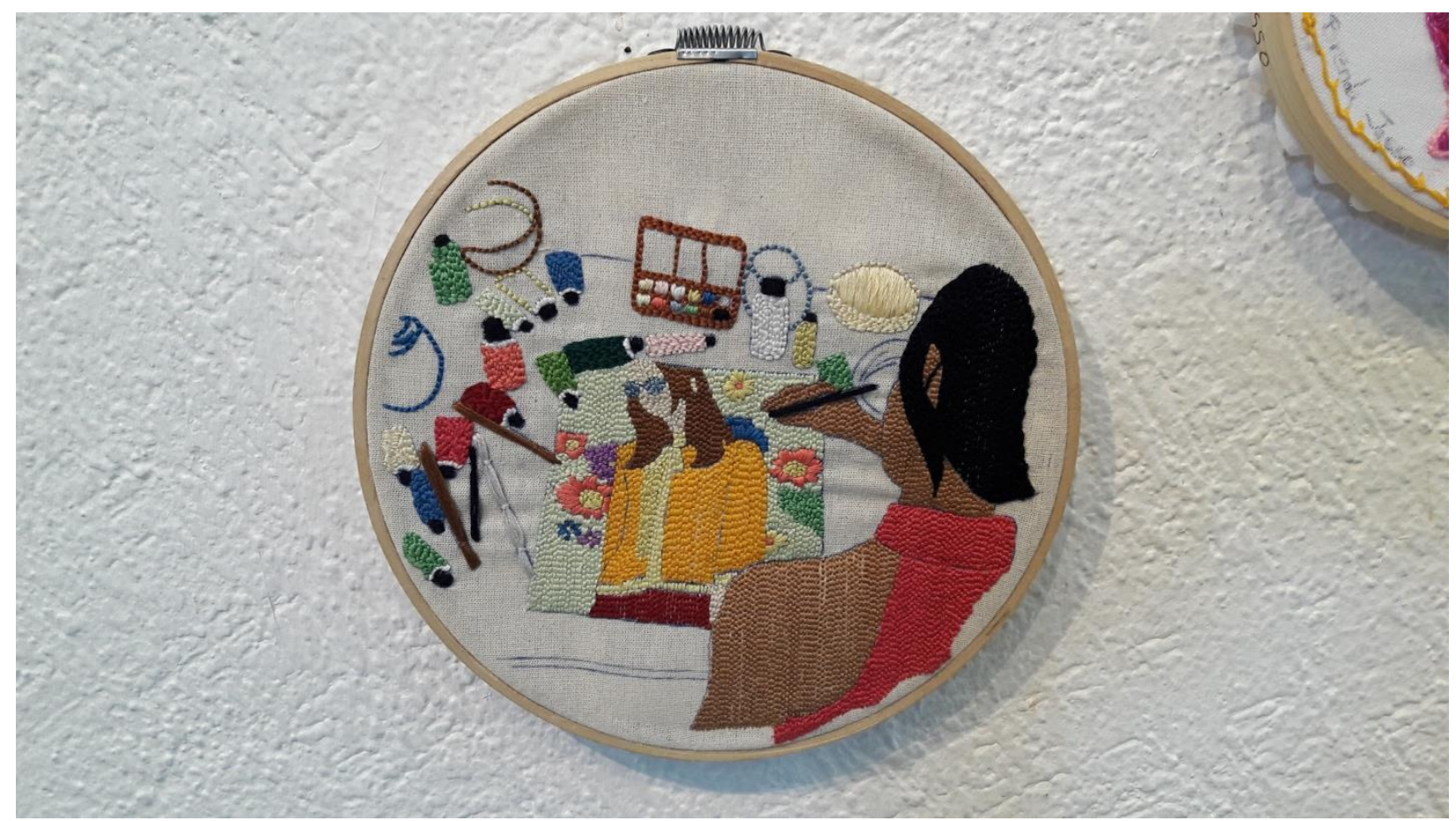

Figura 05. Bordado de alumna, proyecto personal.

Fuente: archivo personal de los investigadores, 2017. 
Uno de nuestros propósitos durante las sesiones fue propiciar el conocimiento, valoración y dominio de algunas técnicas básicas del tejido, así como, sus características. Esto con la intención de incentivar en cada una, la creación y la experimentación de nuevas técnicas, formas, texturas y diseños en textiles. Como hemos podido observar en los testimonios, la participación en el taller propició el aprendizaje de conocimientos, actitudes y valores que fueron construidos a través de la experiencia directa con los materiales textiles. Gradualmente, vino su resignificación mediante actividades sugeridas durante las clases y las propuestas creativas de los y las estudiantes. Para cada nuevo patrón aprendido, tales como el enlazado, tafetán (o 'uno a uno' ), trenzado, kilim4 , columnas, flecos, embarrilado, se observaba que los y las estudiantes lograban también la libertad para decidir cuáles materiales usarían para crear sus proyectos, procurando ir más allá de los estambres de lana o sintéticos. El dominio técnico permitía la libertad expresiva, donde podían plasmar con más precisión, las ideas que tenían sobre el tejido.

Por otro lado, hemos observado que el contacto directo con el quehacer artesanal propiciaba la comprensión de los procesos de hechura y una sutil revalorización de la mirada sobre esta práctica. Si esta nueva mirada será para toda la vida, es difícil saberlo. Pero al menos podemos decir, que con base en lo trabajado en el taller de telares, que cuando se conoce cómo se hace una artesanía, las personas se sensibilizan y algunas entienden la importancia de su valor, de lo que cuesta, de lo que representa para una comunidad o para un artesano o artesana en particular el oficio que realiza.

Lo anterior expresa la aplicación de la Investigación Basada en la Artes, de acuerdo con Hernández (2008, p. 93): "utiliza elementos artísticos y estéticos como forma de pensar desde y sobre lenguajes no verbales", es decir, los y las estudiantes empiezan a pensar a partir del contacto directo y de la práctica con los textiles y sus procesos de creación; "busca maneras de mirar y representar la experiencia sin para ello, perseguir las certezas, sino el realce de perspectivas distintas", lo que podríamos traducir como el fomento en la creación de diseños propios, a partir de sus biografías, el trabajo en equipo, desarrollo de la capacidad de mirar y de pensar sobre lo mirado; (la problematización del trabajo artesanal en México, la separación de actividades y oficios por género, los estereotipos en cuanto a la práctica de un oficio artesanal, entre otros), y "trata de desvelar aquello de lo que no se habla" (sobre la importancia de resignificar miradas, prejuicios y confusiones en torno a la práctica de los oficios artesanales y textiles en México).

\footnotetext{
${ }^{4}$ Es un tipo de punto de origen turco que permite crear formas geométricas en el tejido en telar de clavos. 
Pensamos que nuestro trabajo como docentes en el taller de telares no es solo enseñar algunas técnicas para que los y las estudiantes pasen el tiempo, sino provocarles a desacomodar posibles miradas que hayan construido sobre las artesanías en México. Por este motivo, conocer el lenguaje o sus ramas (cestería, cerámica, cartonería, textiles, entre otros), se relaciona directamente con el conocimiento y comprensión de un modo de vida: lo artesanal. Es decir, todo lo que conlleva el proceso de creación de una artesanía, sus tiempos, sus ritmos, sus técnicas, las leyendas e historias que mueven el imaginario de los y las artesanas que transforman la materia que encuentran en su localidad y contexto. Aprender con las manos y mediante el contacto de la materia con nuestro cuerpo, también nos enseña desde el conocimiento empírico.

Dice Chloe Sayer que: "las escuelas gubernamentales deberían respetar las tradiciones artesanales locales e invitar a los maestros artesanos para enseñar en el contexto del salón" (1998, p. 64). Pese el trabajo que hemos estado realizando en la institución, pensamos que este vínculo entre artesanas y artesanos y el contexto universitario (nos referimos específicamente a la institución donde laboramos) aún está por construirse. Concretamente, no hay una vinculación formal entre la Universidad de Guanajuato, Campus León, con artesanos y artesanas de la región. De modo que la vinculación se hace más por iniciativas de profesores y profesoras, alumnos y alumnas, pero consisten en esfuerzos aislados, es decir: va depender del tipo de actividad que se planee durante el semestre (si es una exposición, coloquio, celebración), donde ocasionalmente se invitan a artesanos y artesanas para que expongan y vendan sus trabajos en la institución. Pero muy pocas veces, se piensa en ellos y ellas como actores que también podrían hablar y enseñar sobre lo que hacen.

\section{Consideraciones finales}

México es reconocido mundialmente como uno de los países con mayor producción artesanal, quedando solamente atrás de India y China (HEL HERALDO DE MÉXICO, 2017). Esto se traduce en que aproximadamente 12 millones de personas se dedican a la creación artesanal, siendo la mayoría, mujeres. También representa el $10.3 \%$ de la población económicamente activa en el país trabajando en la producción artesanal ya sea de tiempo completo o parcial (ENCUNM, 2012). Por tal motivo, nos parece importante que las artesanías y sus ramas sean un constante tema de debate, investigación y valoración por parte de la población mexicana. Su invisibilización o descaso ha sido tan reiterada, que apenas en el 2020, se planea realizar el primer censo especializado para identificar los perfiles de artesanos y artesanas tradicionales de este país (EL MILENIO, 2018).

Revista Digital do LAV - Santa Maria - vol. 12, n. 1, p. 38 - 54 - jan./abr. 2019 ISSN 1983 - 7348 http://dx.doi.org/10.5902/1983734837114 
Como hemos podido observar a lo largo de este documento, aunque México sea un país con una gran riqueza cultural y artística, eso no se traduce necesariamente, en el reconocimiento, apreciación y valorización de sus tradiciones y costumbres por parte de los estudiantes y de la sociedad en general. De tal modo, el mayor objetivo de nuestras clases ha sido concienciar a los y las estudiantes sobre la importancia del trabajo artesanal mexicano (y de otros contextos también) y, así, tornar su mirada un poco más receptiva y abierta a las diferentes manifestaciones artísticas y a las culturas estéticas de este país. También hemos buscado problematizar las categorías que definen las fronteras del arte y de las artesanías a fin de repensar sobre esta práctica como una actividad artística que comunica y que fortalece las tradiciones locales, las costumbres e historias de nuestros pueblos. Además de producir objetos útiles, las artesanías también presentan posibilidades expresivas y artísticas.

En este sentido, cuando conocemos algo, miramos los fenómenos de modo distinto; cuando miramos de otra manera, significamos más intensamente las experiencias y damos sentido a otras que nos pasaban desapercibidas. Al conocer el arte textil, ya sea el tradicional o el contemporáneo, así como sus procesos de creación, nos permite transformar la percepción que tenemos al respecto de la cosa creada. Al examinarlo lentamente, podemos saborear sus cualidades y así reconocer tanto sus valores, como sus diferencias y similitudes en diferentes culturas.

\section{Referencias}

BARBOSA, A.M. Arte y Cultura. In: Encuentro Nacional sobre Arte y Diversidad

Cultural. Lima, Perú: Facultad de Arte de la Pontificia Universidad Católica de Perú, 2009.

BOVÍSIO, M. Algo más sobre una vieja cuestión: "arte vs artesanía". Buenos Aires, Argentina: FIAAR, 2002.

CHALMERS, G. Arte, Educación y Diversidad Cultural. Barcelona, España: Paidós Arte y Educación, 2003.

DEL CARPIO, P. S. Entre el textil y el ámbar. Funciones psicosociales del trabajo artesanal de artesanos tsotsiles de La Ilusión, Chiapas, México. Athenea Digital. Revista de Pensamiento e Investigación Social, v.12, p. 185-198, 2012. Disponible en: http://atheneadigital.net/article/view/v12-n2-delcarpio/1015-pdf-es. Acceso en: 2 feb. 2018.

Revista Digital do LAV - Santa Maria - vol. 12, n. 1, p. 38 - 54 - jan./abr. 2019 ISSN 1983 - 7348 http://dx.doi.org/10.5902/1983734837114 
EL HERALDO DE MÉXICO. México, tercer lugar a nivel mundial en la producción de artesanías. 2017. Disponible en: https://heraldodemexico.com.mx/estados/mexicotercer-lugar-a-nivel-mundial-en-elaborar-artesanias/. Acceso en: 20 feb. 2019.

EL MILENIO. Primer censo nacional de artesanos, para 2020. 2018. Disponible en: https://www.milenio.com/negocios/primer-censo-nacional-de-artesanos-para-2020. Acceso en: 20 feb. 2019.

ENCUESTO NACIONAL DE CONSUMO CULTURAL. 2012. Disponible en: https://www.gob.mx/fonart/documentos/las-artesanias-en-la-encuesta-nacional-deconsumo-cultural-de-mexico-enccum. Acceso en: 30 jan. 2019.

FREITAG, V. Memorias del oficio artesanal: un estudio con familias de artesanos de Tonalá, Jalisco. Tesis (Doctorado en Ciencias Sociales). Centro de Investigaciones Y Estudios Superiores - CIESAS-Guadalajara, México, 2012.

GREENHALGH, P. The history of the craft. In: Peter Dormer (Ed.), The culture of craft, Manchester: Manchester University Press, 1997, p. 20-52.

HERNÁNDEZ, F. La investigación basada en las artes. Propuestas para repensar la investigación en educación. Revista Educatio Siglo XXI, v. 26, p. 85-118, 2008.

LANAU, D.; PASCUAL, A. El arte es una forma de hacer (no una cosa que se hace). Reflexiones a partir de una conversación de Luis Camnitzer y María Acaso. Madrid: Los Libros de la Catarata, 2018.

MARÍN VIADEL, R. La investigación en Educación Artística. Revista Educatio Siglo XXI, v. 29, n. 1, p. 211-230, 2011.

NOVELO, V. "La fuerza del trabajo mexicano, protagonista ¿permanente? de la industria". Revista Alteridades, v.18, n. 35, p. 117-126, 2009.

SAYER, C. La misión de educar. Artes de México: Arte Popular Museo Ruth Lechuga, v. 42, p. 63-66, 1998.

TUROK, M. "El artesanado tradicional. Disyuntivas para su sobrevivencia". In: Salvador Pérez Ramírez (Ed.), Artesanías y Saberes Tradicionales. Zamora, México: El Colegio de Michoacán, 2015. p. 77-97. 
' Licenciada em Desenho e Plástica; Mestre em Educação/UFSM; Doutora em Ciências Sociais/CIESAS-Occidente. Professora da Universidad de Guanajuato, Campus León, México

ii Es doctorando en el Programa de Posgrado Interinstitucional en Arte y Cultura y colabora en el proyecto de investigación. Desarrolla un estudio sobre los usos discursivos de la artesanía en México.Licenciado en Artes Plásticas y Maestro en Estudios del Discurso por la Universidad Michoacana San Nicolás Hidalgo, Morelia, Michoacán.

Como citar esse artigo:

FREITAG, Vanessa; CASIMIRO, Jorge Alberto Custodio. Oficios artesanales resignificados a través del proceso creativo con el lenguaje textil: una experiencia en la Educación Superior. Revista Digital do LAV, Santa Maria: UFSM, v. 12, n. 1, p. 34-54, jan./abr. 2019. 